\title{
artigo
}

Macêdo, A.G.A.O.; Parente, F.L.; Freitas, C.A.S.L.; Oliveira, C.M.;

Utilização dos indicadores de saúde no processo de gerenciamento hospitalar

\section{Utilização dos indicadores de saúde no processo de gerenciamento hospitalar}

\author{
Use of health indicators in the hospital management process \\ Uso de indicadores de salud en el proceso de gestión hospitalaria
}

\begin{abstract}
RESUMO
Objetivo: Analisar a utilização dos indicadores de saúde no processo de gerenciamento hospitalar. Método: Pesquisa bibliográfica, tipo revisão da literatura. Realizada através da busca em três bases de dados: Literatura Latino-americana e do Caribe; Medical Literature Analysis and Retrieval System Online e Banco de Dados em Enfermagem (BDENF), por artigos publicados nos anos de 2010 a 2018, no idioma português. Foram utilizados os descritores combinados: "indicadores de gestão", AND "indicadores de qualidade em assistência à saúde" AND "administração hospitalar". Resultados: Os estudos se estruturam em três eixos: 1. Avaliação dos indicadores de saúde nos hospitais; 2. Percepção dos gestores; e 3. Inovação na análise dos indicadores. Verificou-se que erros no preenchimento de registros hospitalares ou a ausência de informações, comprometem a veracidade da informação. Conclusão: Os indicadores de saúde cumprem papel determinante ao traçar um perfil diagnóstico da situação de saúde no processo de gerenciamento hospitalar.
\end{abstract}

DESCRITORES: Indicadores de gestão; Indicadores de qualidade em assistência à saúde; Administração hospitalar.

\section{ABSTRACT}

Objective: To analyze the use of health indicators in the hospital management process. Method: Bibliographic search, such as literature review. Conducted by searching three databases: Latin American and Caribbean Literature; System of Analysis and Recovery of Online Medical Literature and Database in Nursing (BDENF), for articles published in the years 2010 to 2018, in Portuguese. The combined descriptors were used: "management indicators", AND "health care quality indicators" AND "hospital administration". Results: The studies are structured in three axes: 1. Evaluation of health indicators in hospitals; 2. Perception of managers; and 3. Innovation in the analysis of indicators. It was found that errors in filling out hospital records or the absence of information, compromise the veracity of the information. Conclusion: Health indicators play a decisive role in drawing a diagnostic profile of the health situation in the hospital management process.

DESCRIPTORS: Management indicators; Quality indicators in health care; Hospital administration.

\section{RESUMEN}

Objetivo: Analizar el uso de indicadores de salud en el proceso de gestión hospitalaria. Método: Búsqueda bibliográfica, como revisión de la literatura. Realizado mediante la búsqueda de tres bases de datos: Literatura latinoamericana y caribeña; Sistema de Análisis y Recuperación de Literatura Médica en Línea y Base de Datos en Enfermería (BDENF), para artículos publicados en los años 2010 a 2018, en portugués. Se utilizaron los descriptores combinados: "indicadores de gestión", "indicadores de calidad de la atención sanitaria" Y "administración hospitalaria". Resultados: Los estudios se estructuran en tres ejes: 1. Evaluación de indicadores de salud en hospitales; 2 . Percepción de los gerentes; y 3 . Innovación en el análisis de indicadores. Se encontró que los errores en el llenado de los registros hospitalarios o la ausencia de información comprometen la veracidad de la información. Conclusión: Los indicadores de salud juegan un papel decisivo en la elaboración de un perfil diagnóstico de la situación de salud en el proceso de gestión hospitalaria.

DESCRIPTORES: Indicadores de gestión; Indicadores de calidad en salud; Administración hospitalaria.

RECEBIDO EM: 16/03/2021 APROVADO EM: 31/03/2021

\section{Antônia Gabriela Aragão de Oliveira Macêdo}

Enfermeira. Discente do Mestrado Acadêmico em Saúde da Família da Universidade Federal do Ceará (UFC).

ORCID: 0000-0002-7366-2043 


\section{Fabiene Lima Parente}

Mestre em Ensino na Saúde pela Universidade Estadual do Ceará (UECE). Docente da Faculdade Alencarina de Sobral (FAL). ORCID: 0000-0002-6423-3909

\section{Cibelly Aliny Siqueira Lima Freitas}

Doutora em Enfermagem pela Universidade Federal do Ceará (UFC). Docente do Curso de Enfermagem da Universidade Estadual Vale do Acaraú (UVA).

ORCID: 0000-0002-0585-5345

\section{Camila Martins de Oliveira}

Mestre em Saúde da Família pela Universidade Federal do Ceará (UFC). ORCID: 0000-0003-1592-0013

\section{INTRODUÇÃO}

0 s sistemas de informação podem ser definidos como um conjunto de componentes interrelacionados que coletam dados, com a finalidade de disponibilizar informação para análise, compreensão dos principais problemas de saúde da população, norteando para tomada de decisões ${ }^{(1)}$.

Para um andamento eficaz dos sistemas de informação em saúde deve-se assegurar a produção de informação confiável e oportuna a respeito do estado de saúde da população, seus determinantes e o desempenho do sistema de saúde, porém deve-se também executar análises para direcionar as atividades nos diversos blocos do sistema. Da mesma forma, as estatísticas de saúde precisam permitir que os gestores, em todos os níveis do sistema, reconheçam avanços, problemas e necessidades, tomem decisões baseadas em evidências sobre políticas de saúde. Neste processo, o uso de indicadores de saúde torna mais simples a atividade do gestor ${ }^{(2)}$.

Os indicadores têm como finalidade orientar na tomada de decisão, pois indicam o momento, a hora, o tempo e o lugar para que se desenvolva uma ação; ou seja, o indicador é o índice critico capaz de nortear a tomada de decisão em prol das evidencias ou providencias ${ }^{(3)}$.

Os indicadores têm como propósito fornecer dados para analisar os serviços de saúde, facilitando e permitindo o supervisionamento de objetivos e metas em saúde, e com isso a possibilidade de acompanhar a qualidade do atendimento, instigando o fortalecimento da capacidade de análi- se das equipes de saúde, identificando as oportunidades de melhoria, mudanças positivas no serviço e o desenvolvimento de sistemas de informação de saúde intercomunicados $^{(4)}$.

A gestão da saúde exige a tomada de decisões de elevada responsabilidade e relevância social. As informações a partir dos indicadores de saúde fornecem o embasamento necessário ao planejamento, à execução e à avaliação das açôes realizadas, na medida em que propiciam o conhecimento sobre aspectos relevantes da população, reduzem o grau de incerteza sobre sua situação de saúde e apoiam a busca de possíveis soluções e providências ${ }^{(2)}$.

Vieira, Detoni e Braum ${ }^{(5)}$ em seu estudo "Indicadores de Qualidade em uma Unidade Hospitalar" expõem que o uso de indicadores para avaliar os serviços de saúde é uma das bases do sucesso para garantir qualidade nos serviços hospitalares, deste modo os indicadores se tornam mais uma ferramenta para as instituições de saúde melhorarem a assistência a população.

Tendo em vista as considerações anteriores o interesse por esse estudo surgiu pela percepção de que os indicadores de saúde representam mais do que um conjunto de dados, pois adicionam valores aos mesmos, convertendo-os em informações a serem utilizadas pelos gestores. Deste modo, este estudo poderá contribuir para o conhecimento em gestão hospitalar, possibilitando o conhecimento das informações produzidas dentro da assistência, identificação das fragilidades e pontos positivos nos serviços de saúde, subsidiando as equipes e gestores na reflexão para a tomada de decisões estratégicas e eficientes com vistas para melhoria permanente $\mathrm{e}$ continua na qualidade assistencial.

Nesta perspectiva este estudo propõe analisar a utilização dos indicadores de saúde no processo de gerenciamento hospitalar.

\section{MÉTODO}

Trata-se de uma pesquisa bibliográfica, que consiste em um procedimento metodológico importante na produção do conhecimento científico capaz de gerar, especialmente em temas pouco explorados, a postulação de hipóteses ou interpretações que servirão de ponto de partida para outras pesquisas ${ }^{(6)}$, do tipo revisão da literatura.

A pesquisa foi realizada no período de julho a novembro de 2018. Delimitado o recorte temporal para a pesquisa de artigos dos anos de 2010 a 2018, através da busca nos periódicos científicos, disponíveis em três bases de dados: Literatura Latino-americana e do Caribe (LILACS); Medical Literature Analysis and Retrieval System Online (MEDLINE) e Banco de Dados em Enfermagem (BDENF), encontrados na Biblioteca Virtual em Saúde (BVS), no idioma português. Foram utilizados os descritores combinados: "indicadores de gestão", AND "indicadores de qualidade em assistência à saúde" AND "administração hospitalar".

Os critérios de inclusão empregados para seleção dos artigos foram: estudos disponíveis eletronicamente que abordassem na temática o uso de indicadores de saúde no processo de gerenciamento hospitalar. E como critério de exclusão os 


\section{artigo}

Macêdo, A.G.A.O.; Parente, F.L.; Freitas, C.A.S.L.; Oliveira, C.M.;

Utilização dos indicadores de saúde no processo de gerenciamento hospitalar

artigos duplicados e que não atenderam ao objetivo do estudo.

Após a seleção dos artigos que respondessem os critérios de elegibilidade previamente definidos, foram seguidos os seguintes passos: leitura exploratória, leitura seletiva, escolha do material que contemplasse os objetivos deste estudo, análise dos textos, realização de leitura interpretativa. Os achados foram analisados conforme a questão de pesquisa, apresentados por meio de quadro, sintetizados de forma narrativa e discutidos criticamente com a literatura pertinente

\section{RESULTADOS}

A quantidade de artigos completos encontrados por meio dos descritores: indicadores de gestão, indicadores de qualidade em assistência à saúde e administração hospitalar, disponíveis nos Descritores em Ciências da Saúde (DeCS). Resultou no total de 39 artigos, na seguinte disposição: LILACS (26), MEDLINE (04) e BDENF (09).

Ao considerar apenas os estudos publicados entre os anos de 2010 e 2018, foram eliminados 12 estudos. Após a análise de títulos e resumos e aplicação dos critérios de inclusão e exclusão, ficaram 27 estudos. No entanto, 06 não atendiam aos critérios propostos e, finalmente, 21 resumos foram aceitos para que seus artigos completos fossem lidos posteriormente. Destes, 5 artigos foram incluídos para a revisão.

Para facilitar a interpretação dos dados, os artigos incluídos foram agrupados em três eixos, conforme os assuntos mais discutidos. No quadro 1, estão especificados o eixo, o título do artigo, o tipo de estudo e os desfechos de cada estudo.

Dos artigos analisados, a maioria apresentou como temática principal o eixo 1, que versa sobre avaliação dos indicadores de saúde. No eixo 2 , retrata acerca da percepção dos gestores, foi encontrado apenas um artigo. Em seguida no eixo 3, que faz alusão às inovaçóes na análise dos indicadores, nota-se que também houve poucas evidências científicas nesse aspecto, sendo delimitado somente um estudo.

Em relação aos tipos de estudos, houve predominância da pesquisa descritiva com abordagem qualitativa. Em menor proporção aparece pesquisa quantitativa $\mathrm{e}$ resenha crítica.

\section{DISCUSSÃO}

No tocante aos resultados evidenciados, observa-se que a maioria dos estudos seguiu um sistema metódico, ao realizar a análise seguindo passos previamente estabelecidos, discriminados por: seleção das variáveis, a construção dos indicadores simples; o estabelecimento de valor para os indicadores simples; a elaboração dos

\section{Quadro 1. Identificação dos artigos selecionados que versam sobre os indicadores de saúde.}

\begin{tabular}{|c|c|c|c|c|}
\hline EIXO & FONTE/ ANO & TITULO & TIPO DE ESTUDO & DESFECHO \\
\hline \multirow{3}{*}{$\begin{array}{l}\text { 1. Avaliação } \\
\text { dos indicado- } \\
\text { res de saúde } \\
\text { nos hospitais }\end{array}$} & $\begin{array}{l}\text { Medicina (Ribeirão } \\
\text { Preto) }^{\left({ }^{(7)}\right.} 2017\end{array}$ & $\begin{array}{l}\text { Descrição de prática } \\
\text { para a gestão da far- } \\
\text { mácia hospitalar. }\end{array}$ & $\begin{array}{l}\text { Trata-se de um estudo } \\
\text { descritivo, longitudinal, } \\
\text { sobre a evolução do indi- } \\
\text { cador chave Taxa de Falta } \\
\text { de Medicamentos Padro- } \\
\text { nizados na assistência } \\
\text { do paciente internado, no } \\
\text { período de março de } 2004 \\
\text { a dezembro de } 2013 \text {. }\end{array}$ & $\begin{array}{l}\text { A abordagem de gestão baseada em } \\
\text { processos foi eficaz para a farmácia } \\
\text { hospitalar. A premissa adotada é que } \\
\text { as mudanças administrativas (inter- } \\
\text { ferências), com foco na melhoria dos } \\
\text { processos e seleção e acompanhamento } \\
\text { de indicadores, têm influência sobre os } \\
\text { processos, reduzindo a variabilidade e } \\
\text { melhoria da qualidade. }\end{array}$ \\
\hline & $\begin{array}{c}\text { Rev Bras Enferm }{ }^{(8)} \\
2016\end{array}$ & $\begin{array}{l}\text { Análise de indicadores } \\
\text { gerenciais e } \\
\text { assistenciais após } \\
\text { adequação de pessoal } \\
\text { de enfermagem. }\end{array}$ & $\begin{array}{c}\text { Estudo descritivo, } \\
\text { retrospectivo com dados } \\
\text { obtidos dos sistemas de } \\
\text { registros informatizados } \\
\text { de um hospital } \\
\text { universitário do Sul do } \\
\text { Brasil. }\end{array}$ & $\begin{array}{l}\text { A adequação do quantitativo de } \\
\text { pessoal repercutiu positivamente nos } \\
\text { indicadores gerenciais e assistenciais, } \\
\text { e contribuiu para qualificar o cuidado e } \\
\text { melhorar as condições de trabalho da } \\
\text { equipe de enfermagem. }\end{array}$ \\
\hline & $\begin{array}{l}\text { CuidArte Enfermagem } \\
\text { (9) } 2016\end{array}$ & $\begin{array}{l}\text { Critérios da avaliação } \\
\text { do serviço de } \\
\text { enfermagem nos } \\
\text { programas de } \\
\text { acreditação hospitalar: } \\
\text { uma análise crítica. }\end{array}$ & $\begin{array}{l}\text { Resenha crítica, realizada } \\
\text { após a seleção de texto } \\
\text { sobre acreditação. }\end{array}$ & $\begin{array}{l}\text { Observa-se maior ênfase na área } \\
\text { administrativa, no entanto, pode- } \\
\text { se afirmar que todas as áreas } \\
\text { estão interligadas e necessitam de } \\
\text { investimento para que se obtenha a } \\
\text { excelência do serviço. }\end{array}$ \\
\hline $\begin{array}{l}\text { 2. Percepção } \\
\text { dos gestores }\end{array}$ & Saúde Soc. ${ }^{(2)} 2015$ & $\begin{array}{l}\text { Percepção dos } \\
\text { gestores sobre o uso } \\
\text { de indicadores nos } \\
\text { serviços de saúde. }\end{array}$ & $\begin{array}{l}\text { Trata-se de um estudo } \\
\text { descritivo e exploratório } \\
\text { com abordagem de } \\
\text { análise qualitativa. }\end{array}$ & $\begin{array}{l}\text { Verificou-se que, em maioria, os } \\
\text { gestores entrevistados não se } \\
\text { apropriam dos indicadores de saúde } \\
\text { para suas ações gerenciais. }\end{array}$ \\
\hline
\end{tabular}




\begin{tabular}{|c|c|c|c|c|}
\hline $\begin{array}{l}\text { 3. Inovação } \\
\text { na análise dos } \\
\text { indicadores }\end{array}$ & Physis ${ }^{(10)} 2013$ & $\begin{array}{c}\text { Démarche } \\
\text { Estratégica: modo } \\
\text { inovador e eficaz de } \\
\text { análise da missão } \\
\text { institucional. }\end{array}$ & $\begin{array}{l}\text { O estudo foi realizado } \\
\text { no período de junho a } \\
\text { setembro de } 2009 \text { em } \\
\text { uma maternidade pública } \\
\text { estadual, utilizado como } \\
\text { método de análise da } \\
\text { gestão e como avaliação } \\
\text { da instituição hospitalar } \\
\text { numa perspectiva de rede } \\
\text { coordenada de serviços. }\end{array}$ & $\begin{array}{l}\text { O estudo mostrou a potencialidade } \\
\text { do enfoque como um instrumento } \\
\text { de diagnóstico situacional e de } \\
\text { ferramenta de gestão hospitalar para } \\
\text { rever a missão institucional na busca } \\
\text { de qualidade e solidariedade dentro } \\
\text { da rede e no compromisso com o } \\
\text { atendimento ao usuário de forma } \\
\text { universal e equânime. Contudo, os } \\
\text { compromissos expressos na síntese } \\
\text { propositiva devem ser constantemente } \\
\text { revistos e repactuados entre os atores, } \\
\text { para alcançar mudanças institucionais } \\
\text { permanentes. }\end{array}$ \\
\hline
\end{tabular}

indicadores compostos; a valoração dos indicadores compostos; o desenvolvimento do índice de completude e, por fim, a definição do valor para o índice.

Entretanto, alguns optaram pela aplicação da teoria de Donabedian, cujos indicadores englobam a estrutura, o processo e os resultados. O indicador de avaliação do estrutura foi o mais evidenciado nas produções, um dos aspectos avaliados trata-se do índice de treinamento dos profissionais ${ }^{(11)}$.

Ainda na perspectiva de avaliação dos indicadores nos hospitais, cabe destacar a educação permanente como elemento fundamental para a manutenção do padrão de qualidade do serviço. A equipe precisa ser treinada e atualizada continuamente, em vista de melhorar a assistência. $\mathrm{O}$ enfermeiro, em especial, que por vezes é responsável pelo gerenciamento da equipe, necessita do envolvimento constante em programas de treinamento para incentivo dos demais membros ${ }^{(12)}$.

Essa evidência corrobora com estudo produzido por Kurcgant ${ }^{(13)}$ ao afirmar em uma pesquisa na área de gerenciamento que o enfermeiro possui essa habilidade de transformar a prática a partir do conhecimento, valores e olhar holísticos obtidos durante sua formação profissional.

A qualidade assistencial de saúde é definida como uma associação de boas práticas pautadas em teorias atualizadas, que visa a produção de serviços condizentes com as necessidades e particularidades dos pacientes. A organização do cuidado pode ser auxiliada pela aplicação da Sistematiza- ção da Assistência de Enfermagem (SAE) que faz essa união entre teoria e prática em suas ações ${ }^{(14)}$.

Nesse contexto, os indicadores de saúde, contribuem de forma positiva com os diversos serviços que o utilizam, ao permitir a identificação dos problemas que precisam ser priorizados e auxiliar no estabelecimento de metas a serem alcançadas. No entanto, para que se tenham dados precisos é necessário efetuar o registro corretamente. Para que um indicador seja considerado bom, deve-se atender a algumas características a fim de garantir maior confiabilidade dos dados. Sendo as mesmas expressas por objetividade, ao trazer informações simples e diretas; simplicidade, de fácil acesso; validade, com capacidade de apontar os problemas; sensibilidade, apto a perceber os detalhes e possuir baixo custo ${ }^{(15)}$.

Verificou-se em alguns estudos, que erros no preenchimento de registros ou a ausência de informações, são fatores que comprometem a integridade dos resultados e a veracidade da informação. Contribuindo assim, para a subnotificação de agravos à saúde e pouco investimento destinado à prevenção de adversidades, o que reflete na permanência dos riscos ${ }^{(2)}$.

Constata-se que dentre as principais causas relacionadas às inadequações no preenchimento de registro dos indicadores, destacam-se as características singulares do sujeito que remete à falta de atenção, falta de tempo e pouco conhecimento sobre o sistema. Ademais, também estão associadas ao perfil estrutural das unidades e gerencial, como a deficiência de recursos humanos, que é menor do que a demanda ${ }^{(16)}$.

Neste sentido, estudo realizado no âmbito hospitalar ${ }^{(17)}$ demonstra que a gestão documental se mostra como uma ferramenta capaz de aprimorar a utilização de documentos de forma qualificada, além de diminuir o tempo na recriação ou busca de documentos perdidos, destinando assim esses recursos para a prestação de outros serviços destinados a assistência ao paciente.

Em conformidade com o estudo desenvolvido por Silveira ${ }^{(16)}$, que versa sobre a opinião de enfermeiros de instituições hospitalares acerca da utilização dos indicadores de qualidade da assistência de enfermagem, realizado em dois hospitais filantrópicos do interior de Minas Gerais, foi possível delimitar alguns impasses causados pelo desconhecimento da aplicação dos indicadores em saúde.

Já em relação a percepção dos gestores, o repasse de informações sobre o processo de trabalho entre os funcionários e a comunicação, pode ser apontado como um elemento desafiador no trabalho, uma vez que as informações, podem não chegar a todos de maneira uniforme. Ressalta-se a importância do profissional satisfeito e motivado pela aquisição de metas e indicadores desejados, o que tende a produzir mais e melhor, tendo impacto na melhoria do serviço para o paciente ${ }^{(18,19)}$.

Na perspectiva da inovação da análise dos indicadores a Démarche Estratégica evidencia um modo eficaz para proble- 


\section{artigo}

Macêdo, A.G.A.O.; Parente, F.L.; Freitas, C.A.S.L.; Oliveira, C.M.

matização dos processos de trabalho e para a análise da gestão hospitalar, com a possibilidade de apontar estratégias de ação validadas pelos diferentes atores. $\mathrm{O}$ método pode ser considerado um modo inovador e efetivo para produzir equipes de saúde mais solidárias e corresponsáveis com movimentos de mudança das práticas $^{(10)}$.

Ressalta-se a importância da compreensão dos indicadores de saúde pelos gestores e equipe, o que facilita a análise dos resultados e incorporação das informações adquiridas, a fim de gerar mudanças e aprimoramento conforme as metas determinadas previamente pelas instituições de saúde.

\section{CONCLUSÕES}

Portanto, observa-se que os indicadores de saúde cumprem papel determinante ao traçar um perfil diagnóstico da situação de saúde local e avaliação do atendimento quer seja hospitalar ou ambulatorial. Dessa forma, auxiliando na identificação precoce dos problemas.

Observa-se que as instituições de saúde e gestores, precisam assegurar uma assistência com maior qualidade à população, a partir do incentivo a educação permanente, ao disponibilizar cursos para o aprimoramento e atualização dos conhecimentos, a fim de gerar impacto que culmine na mudança do comportamento dos profissionais. Outros aspectos relevantes são a importância do preenchimento correto dos registros para a manutenção da qualidade do gerenciamento e assistência à saúde, e o dimensionamento de pessoal, conforme a demanda de cada setor, a fim de diminuir a sobrecarga e reduzir as chances de erros humanos.

Acredita-se que através dessas medidas possa-se conseguir melhores registros, aumentando o nível de confiabilidade dos indicadores de saúde e consequentemente, ganhando maior relevância nas políticas públicas.

Evidencia-se que a utilização de indicadores de saúde pelos estabelecimentos hospitalares tem apresentado resultados promissores, sendo importante para a tomada de decisão e o planejamento de estratégias para implementação de mudanças. ․

\section{REFERÊNCIAS}

1. Brasil. Ministério da Saúde. Saúde Brasil 2008: 20 anos de Sistema Único de Saúde (SUS) no Brasil. Brasília: Ministério da Saúde; 2009.

2. Lima KWS, Antunes JLF, Silva ZP. Percepção dos gestores sobre o uso de indicadores nos serviços de saúde. Saúde soc. 2015;24(1):61-71.

3. Escobar AL. Epidemiologia \& saúde. Cad. Saúde Pública [Internet]. 1995 [acesso 2018 Set 13];11(1):149-150. Disponivel em: http://www.scielo.br/scielo.php?script=sci_arttext\&pid=S0102-11X1995000100022\&Ing=pt.

4. Caldana G, Gabriel CS, Bernardes A, Évora YDM. Indicadores de Desempenho em serviço de enfermagem hospitalar: revisão integrativa. Rev. Rene, Fortaleza, 2011;12(1):189-197.

5. Vieira DK, Detoni DJ, Braum LMS. Indicadores de Qualidade em uma unidade hospitalar. In: III SEGeT - Simpósio de Excelência em gestão e tecnologia; 2006.

6. Lima TCS, Mioto RCT. Procedimentos metodológicos na construção do conhecimento científico: a pesquisa bibliográfica. Rev. Katál, 2007 [acesso 2018 set 12];10(esp), 37-45. Disponivel em: https://www.scielo.br/pdf/rk/v10nspe/a0410spe.pdf.

7. Pereira LMV, Abramovicius AC, Ungari AQ, Oliveira HBD, Aragon $D C$, Costa AL, Forster AC. Descrição de prática para a gestão da farmácia hospitalar. Medicina (Ribeirão Preto), 2017;50(1):66-75.

8. Quadros DV, Magalhães AMM, Mantovani VM, Rosa DS, Echer IC. Análise de indicadores gerenciais e assistenciais após adequação de pessoal de enfermagem. Rev Bras Enferm. 2016;69(4):684-90.

9. Vilela RPB, Vilela Filho J. Critérios da avaliação do serviço de enfermagem nos programas de acreditação hospitalar: uma análise crítica. CuidArte Enfermagem. 2016;10(2):227-230.

10. Araújo MJD, Artmann E, Andrade MAC. Démarche Estratégica: modo inovador e eficaz de análise da missão institucional. Physis.
2013:23(2):319-343.

11. Donabedian A. Evaluating the quality of medical care. Milbank Mem Fund Q. 1996 [cited 2019 Ago 11];83(4):691-729. Disponível em: DOI: 10.1111/j.1468-0009.2005.00397.x

12. Lara SL. Dimensão ética do gerenciamento em enfermagem [monografia] [internet]. Botucatu: Universidade Estadual Paulista - UNESP; 2010. 54 p. [acesso em 2019 set 13]. Disponível em: https://repositorio.unesp.br/bitstream/handle/11449/119573/ lara_sr_tcc_botfm.pdf?sequence=

13. Kurcgant $P$, Ciampone MHT. A pesquisa na área de gerenciamento em enfermagem no Brasil. Rev Bras Enferm, [Internet]. 2005 [acesso 2019 set 11] ;58(2):161-164. Disponivel em: https:// www.scielo.br/pdf/reben/v58n2/a06.pdf.

14. Mezomo JC. Gestão da qualidade na saúde: princípios básicos. São Paulo: Editora Manole; 2001.

15. Bittar OJNV. Indicadores de qualidade e quantidade em saúde. Rev. de Administração em Saúde, 2001;3(12):21-28.

16. Silveira TVL, Prado Júnior PP, Siman AG, Amaro MOF. Opinião dos enfermeiros sobre a utilização dos indicadores de qualidade na assistência de enfermagem. Rev Gaúcha Enferm, 2015. [acesso 2019 set 12];36(2):82-8. Disponivel em: https://www.scielo.br/ pdf/rgenf/v36n2/pt_1983-1447-rgenf-36-02-00082.pdf.

17. Guimarães GLP, Pereira RS, Paula EJC, Mendes GN, Peixoto MB, Spagnol CA. A importância da gestão documental em unidades de terapia intensiva. Saúde coletiva. 2021;(11)60:4726-4730.

18. Manzo BF, Brito MJM, Correa AR. Implicações do Processo de Acreditação Hospitalar no Cotidiano de profissionais de Saúde. Rev Esc Enferm. 2012;46(2):388-94.

19. Feldman L, Cunha I. Identificação dos critérios de avaliação de resultados do serviço de enfermagem nos programas de acreditação hospitalar. Rev Lat Americ Enferm. 2006;14(4):540-5. 